\title{
Plasma glycated CD59 (gCD59), a novel biomarker for the diagnosis, management and follow up of women with Gestational Diabetes (GDM) - protocol for prospective cohort study
}

D. Bogdanet ${ }^{1,2^{*}}$, PM. O'Shea ${ }^{2}$, J. Halperin ${ }^{3}$ and F. Dunne $e^{1,2}$

\begin{abstract}
Background: The prevalence of Gestational Diabetes (GDM) is rising and with it the number of mothers and children at risk of adverse outcomes. As treatment has been shown to reduce adverse events, it is imperative that we identify all at-risk pregnant women. In Ireland, the national standard of care is selective screening with a 2-hour $75 \mathrm{~g}$ oral glucose tolerance test (OGTT). Aiming for universal screening is of utmost importance but this is difficult given the length, the unfeasibility and impracticability of the OGTT. We aim to assess if the novel biomarker glycated CD59 (gCD59) is a suitable contender for the OGTT in identifying women with GDM.

Methods: In this prospective cohort study, the study participants will be consecutive pregnant women at Galway University Hospital, Galway, Ireland. Samples for the plasma gCD59 biomarker will be taken together with routine bloods at the first antenatal visit, at weeks 24-28 at the time of routine $75 \mathrm{~g}$ OGT, in trimester 3- and 12-weeks post-partum for women with GDM while having their routine post-partum $75 \mathrm{~g}$ OGTT. The constructed database will contain baseline information on each study participant, baseline laboratory data, follow-up laboratory data and pregnancy related outcomes. We aim to recruit a total of 2,000 participants over the project period and with a national GDM prevalence of 12-13\%, we will have 240-260 subjects who meet OGTT criteria for GDM. Following regional prevalence, we expect to have 34-37 women who will develop either diabetes or pre-diabetes in the early post-partum period. The sensitivity and specificity of plasma gCD59 to predict the results of the OGT will be assessed using nonparametric estimates of the receiver operating characteristic (ROC) curves and respective area under the ROC curve (AUROC).
\end{abstract}

Discussion: A body of clinical and experimental evidence supports a link between the complement system, complement regulatory proteins, and the pathogenesis of diabetes complications.

Building on this research, our study plans to look at the plasma gCD59 capacity to classify pregnant women with (Continued on next page)

\footnotetext{
* Correspondence: deliabogdanet@gmail.com

${ }^{1}$ College of Medicine Nursing and Health Sciences, National University of Ireland Galway, Galway, Ireland

2Diabetic Day Centre, Galway University Hospital, Galway, Ireland

Full list of author information is available at the end of the article
}

(c) The Author(s). 2020 Open Access This article is licensed under a Creative Commons Attribution 4.0 International License, which permits use, sharing, adaptation, distribution and reproduction in any medium or format, as long as you give appropriate credit to the original author(s) and the source, provide a link to the Creative Commons licence, and indicate if changes were made. The images or other third party material in this article are included in the article's Creative Commons licence, unless indicated otherwise in a credit line to the material. If material is not included in the article's Creative Commons licence and your intended use is not permitted by statutory regulation or exceeds the permitted use, you will need to obtain permission directly from the copyright holder. To view a copy of this licence, visit http://creativecommons.org/licenses/by/4.0/ The Creative Commons Public Domain Dedication waiver (http://creativecommons.org/publicdomain/zero/1.0/) applies to the data made available in this article, unless otherwise stated in a credit line to the data. 
(Continued from previous page)

normal or abnormal glucose tolerance but also to assess if plasma gCD59 can be used as an early predictor for

GDM, for adverse pregnancy outcomes and/or post-partum glucose intolerance.

Keywords: Gestational diabetes, biomarker, CD59

\section{Background}

Gestational diabetes is a global epidemic that causes adverse maternal and infant outcomes and also identifies the mother-child pair at risk of future diabetes, obesity and cardiovascular disease [1-4]. Treatment of GDM reduces the incidence of adverse outcomes [5-7]. Selective screening for GDM with a $75 \mathrm{~g}$ OGTT is the current Irish national standard of care for all at-risk pregnant women without established diabetes [8]. However, universal screening is the gold standard and advocated by international bodies [9]. Universal screening is difficult to achieve currently because the standard OGTT has to be done fasting, is lengthy, cumbersome for the woman and the health service, and has poor reproducibility [10-12]. Globally researchers are working to identify biomarkers that may replace the OGTT and allow universal screening to become a reality.

Glycated CD59 (gCD59) is one such novel biomarker. CD59 is a complement regulatory protein that protects "self" cells from complement-mediated damage [13, 14]. In diabetes, CD59 is inactivated by non-enzymatic glycation forming gCD59. Plasma gCD59, is a soluble form of CD59 shed from cell membranes. CD59 is a widely distributed membrane-bound inhibitor of the cytolytic membrane attack complex (MAC) of complement[15]. CD59 functions by binding to $\mathrm{C} 8$ and/or $\mathrm{C} 9$ in the nascent MAC and interfering with $\mathrm{C} 9$ membrane insertion and polymerization. The protein, which is present in all cells, is anchored to the external surface of the membrane by a lipid tail. As such it is exposed to the extracellular fluid and the glucose levels in the extracellular fluids. A soluble form of CD59 shed from cell membranes is present in the circulation and urine. Inhibition of the terminal complement cascade is the only known function of CD59 and there is no evidence in the literature that the protein is a marker of inflammation or any other condition, or that significant variations occur in different human diseases.

Pregnancy constitutes a major challenge to the maternal immune system because it requires tolerance of foetal allo-antigens encoded by paternal genes; failure to tolerate, triggers foetal rejection as it does in transplanted organs. The complement system plays a critical role in transplant rejection; similarly, excessive complement activation in the placenta places the foetus at risk for growth restriction or death [16-18].

Therefore, it is not surprising that the foetus is protected from maternal immune responses by an array of mechanisms that include trophoblast expression of key complement regulatory proteins such as decay accelerator factor (DAF), membrane cofactor protein (MCP) and CD59. The key role played by complement and its regulators in pathological pregnancy is highlighted by experimental and clinical data showing that either immunologic maladaptation with activation of complement targeted to the placenta or decreased complement restriction contributes to the imbalance of angiogenic factor that is associated with placental dysfunction in preeclampsia [19-22]. Regarding pregnancy complications of diabetes, it is conceivable that glycationinactivation of placental CD59 increases complementmediated placental damage contributing in part to the multiple complications seen in women with GDM.

Plasma gCD59 can be measured with a highly sensitive and specific enzyme-linked immunosorbent assay (ELISA). Preliminary work in a US population screened by a 2-step glucose challenge test (GCT) followed by $100 \mathrm{~g}$ OGTT using Carpenter \& Coustan criteria, shows promising benefits of plasma gCD59 as a biomarker for GDM [23]. This work demonstrated that plasma gCD59 is 8.5-fold higher in those with a positive compared to a negative GCT and 10fold higher in those who had a positive OGTT following the positive GCT compared to those with a negative OGTT.

The purpose of this prospective study is to examine the validity of plasma gCD59 as a biomarker for the prediction, diagnosis, management and follow up of women with GDM diagnosed using the newer evidence based International Association of the Diabetes and Pregnancy Study Groups (IADPSG) criteria in a 1-step approach using a 75 g OGTT across all BMI categories in an unselected pregnant population. The IADPSG criteria confirm a diagnosis of GDM when fasting glucose is $\geq 5.1 \mathrm{mmol} / \mathrm{L}(92 \mathrm{mg} / \mathrm{dl})$, 1-hour glucose is $\geq 10.0 \mathrm{mmol} / \mathrm{L}(180 \mathrm{mg} / \mathrm{dl})$ or 2-hour glucose is $\geq 0.5 \mathrm{mmol} / \mathrm{L}(153 \mathrm{mg} / \mathrm{dl})$ following a standard $75-\mathrm{g}$ OGTT[1]. As only 1 test and 1 abnormal value are required when using IADPSG criteria, a greater spectrum of women with GDM will be identified. Thus, this study can explore plasma gCD59 in both lower risk and higher risk women.

\section{Methods/Design \\ Study Design \\ Prospective cohort study \\ Objectives}

1 To assess if levels of plasma gCD59 in early pregnancy predict GDM diagnosed by standard of care 75 g OGTT at weeks 24-28. 
2 To assess if plasma gCD59 at weeks 24-28 can replace the $75 \mathrm{~g}$ OGTT as a diagnostic test for GDM.

3 To assess if plasma gCD59 levels in the course of pregnancy help monitor the effectiveness of treatment in women with GDM by examining pregnancy outcomes.

4 To assess if in women with GDM, second trimester (T2) and/or postpartum levels of plasma gCD59 can predict the conversion to diabetes/prediabetes as detected by a $75 \mathrm{~g}$ OGTT at 12 weeks post-partum.

\section{Inclusion criteria}

- Pregnant women 18 years old and over willing and able to provide informed consent.

\section{Exclusion criteria}

- Pregnant women with prior established diabetes.

- Women with concomitant disease or condition that, in the clinical judgment of the investigator, is likely to prevent the subject from conforming to the protocol.

\section{Recruitment}

The study participants will be consecutive pregnant women booking for antenatal care at Galway University Hospital (GUH), Galway, Ireland. The patient information leaflet (PIL) (Supplementary material, 'Patient information leaflet') will be given at the first clinic appointment. The PIL will contain information on the study and a telephone number where the study participant can contact a member of the research team for questions. A member of the research team will explain the study purpose and methodology to the potential study participant. If agreeable, a consent form will be signed, and the first sample of blood will be taken at the time of routine first trimester bloods. The potential study participant will be informed and reassured regarding the low-risk of the study (the samples would be taken at the same time points as routine blood testing) and the confidentiality of the data collected. They will also be informed they can withdraw from the study at any point in time. It will be explained clearly that they can decline to participate without their care being affected in any way.

Samples for the plasma gCD59 biomarker (one additional EDTA bottle) will be taken together with routine bloods at the first antenatal visit, at weeks $24-28$ at the time of routine $75 \mathrm{~g}$ OGTT, in trimester 3- and 12weeks post-partum for women with GDM while having their routine post-partum $75 \mathrm{~g}$ OGTT. This means we will have a sample from each trimester of pregnancy and, for women diagnosed with GDM, a sample 3 months post-partum. This will give us the possibility of looking at the predictability of gCD59 in determining GDM in early pregnancy (the trimester 1 sample), midpregnancy (the trimester 2 sample, same time as the OGTT), determining pregnancy associated complications ( trimester 1, 2 or 3 samples) or determining the development of glucose intolerance post-partum (the 12 weeks post-partum sample). We will also be able to establish gestational reference intervals for gCD59 in women who do not develop GDM. A schematic schedule of enrolment and assessments can be found in Fig 1.

Each gCD59 plasma sample taken will be aliquoted into $2 \times 500 \mu \mathrm{l}$ aliquots and stored at $-80^{\circ} \mathrm{C}$. The samples will be barcoded. One aliquot will be analysed for gCD59 levels while the other aliquot will form our biobank and be stored for future analysis. A clinical database linked to the barcoded samples will be developed by the applicant and pseudo-anonymised. This data will be encrypted, password protected and kept on a secure server. DB, POS, and FD will have the key to deanonymise the data. All laboratory specimens will be identified by a coded ID number to maintain participant confidentiality. To ensure confidentiality, data accessible to team members will be blinded of all identifying participant information.

The constructed database will contain baseline clinical information and laboratory data on each patient, followup laboratory data and pregnancy (maternal and neonatal) related outcomes. We will collect neonatal outcomes ordinarily associated with excess glucose (macrosomia, large for gestational age (LGA), hypoglycaemia, shoulder dystocia and birth injuries, respiratory distress, prematurity, death, NICU admission) maternal outcomes associated with excess glucose (polyhydramnios and post-partum haemorrhage (PPH)), maternal outcomes indicative of the greater vascular risk associated with GDM (pregnancy induced hypertension $(\mathrm{PIH})$, pre-eclampsia (PET)), neonatal outcome often associated with disordered placental vascular architecture e.g. small for gestational age (SGA).

LGA is defined as an infant birth weight greater than or equal to the 90th percentile on a standard growth chart and macrosomia as an infant birth weight greater than or equal to $4000 \mathrm{~g}$. SGA is defined as an infant birth weight less than or equal to the 10th percentile for gestational age on a standard growth chart. Neonatal hypoglycaemia is defined as a plasma glucose level of less than $1.65 \mathrm{mmol} / \mathrm{L}(30 \mathrm{mg} / \mathrm{dL})$ in the first $24 \mathrm{~h}$ of life and less than $2.5 \mathrm{mmol} / \mathrm{L}(45 \mathrm{mg} / \mathrm{dL})$ thereafter.

Prematurity and severe prematurity are defined as a baby born alive before 37 or before 28 completed weeks of pregnancy respectively. Mortality includes stillbirth and neonatal death. Pre-eclampsia is defined as new 


\begin{tabular}{|c|c|c|c|c|c|c|}
\hline \multirow[b]{2}{*}{ TIMEPOINT* } & \multirow{2}{*}{$\frac{\text { Enrolment }}{0}$} & \multicolumn{5}{|c|}{ Post-allocation } \\
\hline & & $t_{1}$ & $t_{2}$ & $t_{3}$ & $t_{4}$ & $t_{5}$ \\
\hline \multirow{2}{*}{$\begin{array}{r}\text { Eligibility } \\
\text { screen } \\
\text { Informed } \\
\text { consent } \\
\end{array}$} & $x$ & & & & & \\
\hline & $x$ & & & & & \\
\hline \multicolumn{7}{|l|}{ ASSESSMENT: } \\
\hline $\begin{array}{r}\text { Routine } \\
\text { bloodwork }\end{array}$ & $x$ & $x$ & $x$ & $x$ & & $x$ \\
\hline OGTT & & & $x$ & & & $x$ \\
\hline $\begin{array}{r}\text { Pregnancy } \\
\text { outcomes } \\
\text { (maternal and } \\
\text { neonatal) }\end{array}$ & & $x$ & $x$ & $x$ & $x$ & \\
\hline
\end{tabular}

Fig. 1 Schedule of enrolment and assessments

onset systolic blood pressure (SBP) of at least $140 \mathrm{mmHg}$ and/or diastolic blood pressure (DBP) of at least $90 \mathrm{mmHg}$ at more than 20 weeks' gestation with proteinuria of greater than $300 \mathrm{mg} /$ day. PIH is defined as new-onset BP at least 140/90 mmHg after 20 weeks gestation with no proteinuria. The decision to proceed with a caesarean delivery is made by the woman's obstetrician. Polyhydramnios is diagnosed when the amniotic fluid index measured is greater than $24 \mathrm{~cm}$ on foetal ultrasound on one or more occasion. Shoulder dystocia is described as foetal shoulders not delivering after the head has emerged from the mother's introitus due to either one or both shoulders becoming impacted against the bones of the maternal pelvis.

This data will be encrypted, and password protected.

\section{Ethics}

Ethical approval has been obtained from the Galway Clinical Research Ethics Committee. This study is conducted in accordance with the guidelines of the Declaration of Helsinki and Good Clinical Practice.

\section{Power calculation and sample size}

The Obstetrics Division of GUH delivers approximately 3,000 infants each year, of which we conservatively expect to recruit 1,000 per year for a total of 2,000 over the project period. With a GDM prevalence of $12-13 \%$ [24], we will have $240-260$ subjects who meet OGTT criteria for GDM in the standard of care testing at week
24-28 and who will have had a plasma gCD59 measurement in the first trimester. If we conservatively assume a dropout rate of $5-10 \%$, we will have $\approx 1,800-1,900$ subjects who will undergo measurement of plasma gCD59 in the first trimester including $216-230$ potentially diagnosed with GDM in the second trimester and having a further plasma gCD59 measurement.

At GUH, approximately 3\% of women with GDM develop T2DM in the early postpartum period, an additional $8 \%$ develop IFG and 4.7\% develop IGT [25]. Based on reported plasma gCD59 values and standard deviation in pregnant women, we estimate that including in the final analysis $\approx 216-230$ (potentially $>240$ ) first trimester samples from women later diagnosed with GDM at weeks 24-28 (as per standard of care practices) will provide $>80 \%$ power to accurately assess the performance of the plasma gCD59 test in early pregnancy to identify women at risk of GDM, with a precision fixed at $0.05 \%$. We will also have $>80 \%$ power to assess the accuracy of plasma gCD59 measured in the second trimester (T2) concomitantly with standard of care OGTT to predict the diagnosis of GDM and to develop an analysis of repeated measurements to assess preliminarily the effectiveness of treatment in women with GDM. Also, in a cohort of 215-230 women with a diagnosis of GDM, at a postpartum conversion rate to type 2 diabetes of $3 \%$ with additional $\approx 13 \%$ converting to glucose intolerance (Impaired Fasting Glucose (IFG) and Impaired Glucose Tolerance (IGT)[25], we expect to have 
34-37 women who will develop either diabetes or prediabetes in the early post-partum period. If postpartum (6-12 weeks) levels of plasma gCD59 are comparable to those reported in a non-pregnant population, we expect to have $80 \%$ power to identify women with GDM who convert to glucose intolerance after delivery.

\section{Statistical Analysis}

Patient characteristics will be described using mean and standard deviations/ medians and interquartile ranges for continuous variables (depending on data distribution) and count proportions for categorical variables. The bivariate baseline characteristics of GDM cases and controls will be compared using binomial/multinomial logistic regression with adjustment for covariates. The sensitivity and specificity of plasma gCD59 to predict the results of the OGTT will be assessed using nonparametric estimates of the receiver operating characteristic (ROC) curves and respective area under the receiver operating characteristic (ROC) curve (AUROC) [26]. The level for significance for all tests conducted will be set at $\alpha=0.05$, with all reported $P$ values as two-tailed. Multiple imputation will be used for missing data.

\section{Study Status}

Recruitment has started February 2019 and it is estimated it will take 18 months for full recruitment.

\section{Discussion}

A body of clinical and experimental evidence supports a link between the complement system, complement regulatory proteins, and the pathogenesis of diabetes complications [27-31].

CD59 is a cell membrane-bound protein. However, a soluble form of CD59 that is shed from cell membranes by phospholipases is present in human blood, urine, saliva, and other body fluids [32-34]. In diabetes, nonenzymatic glycation inactivates the complement inhibitor CD59, forming glycated CD59. By using a highly sensitive and specific ELISA assay, levels of gCD59 were found to be 3- to 4-fold higher in individuals with type 2 diabetes, higher gCD59 concentrations were strongly associated with higher glucose levels after 2-hour oral glucose tolerance tests and the gCD59 level has also been shown to acutely parallel changes in glycaemic control during therapeutic intervention with insulin $[35,36]$. Furthermore, in a population screened using a GCT median gCD59 levels were 8.5-fold higher in the 500 case patients that failed the GCT compared to the control subjects and 10-fold higher in the 127 case patients in whom GDM was diagnosed by the subsequent 3-h OGTT. In a recent retrospective study [37], gCD59 was found to be an accurate biomarker for the early prediction of GDM $($ AUROC $=0.90)$ and also plasma levels of
gGD59 were positively associated with the risk of infant malformations, neonatal hypoglycaemia or delivering an LGA baby (Ref). However, one of the main limitations of this study was that the population recruited was very selective with a BMI $>29 \mathrm{~kg} / \mathrm{m}^{2}$.

Building on this research, our study plans to look at the gCD59 capacity to classify pregnant women with normal or abnormal glucose tolerance as defined by the 2-hour, 75-g OGTT recommended by the IADPSG criteria in an Irish cohort but also assess if gCD59 can be used as an early predictor for GDM, a predictor for adverse pregnancy outcomes and/or post-partum glucose intolerance.

The results will be accessible to physicians and patients and will be published in peer reviewed international literature journals.

\section{Abbreviations}

BMI: Body Mass Index; DAF: Decay Accelerator Factor; DBP: Diastolic Blood Pressure; GDM: Gestational Diabetes; GCT: Glucose Challenge Test;

HSE: Health Service Executive; IADPSG: International Association of the Diabetes and Pregnancy Study Groups; IFG: Impaired Fasting Glucose; IGT: Impaired Glucose Tolerance; LGA: Large for Gestational Age; MAC: Membrane Attack Complex; MCP: Membrane Cofactor Protein; NICU: Neonatal Intensive Care Unit; OGTT: Oral Glucose Tolerance Test; PET: Pre-eclampsia; PIH: Pregnancy Induced Hypertension; PPH: Post-partum haemorrhage; SGA: Small for Gestational Age; SBP: Systolic Blood Pressure

\section{Acknowledgements}

Not applicable.

\section{Authors' contributions \\ DB drafted the manuscript and coordinated manuscript revisions. DB, JH, POS, FD participated in the study design and helped with manuscript revisions. All authors read and approved the final manuscript.}

\section{Funding}

DB is supported by the HRB/Wellcome Trust Irish Clinical Academic Training Programme (ICAT) who is funding this study and who has reviewed and approved the design and methodology of this study. $\mathrm{JH}$ is supported by $\mathrm{NIH}$ grant \# DK1 18528-01A1 and will assist with the laboratory component of this study.

\section{Availability of data and materials \\ The anonymised datasets used and/or analysed during the current study will be available from the corresponding author on reasonable request.}

\section{Ethics approval and consent to participate}

Ethical approval has been obtained from the Galway Clinical Research Ethics Committee. This study is conducted in accordance with the guidelines of the Declaration of Helsinki and Good Clinical Practice. Written consent has been obtained from all study participants to be part of the study.

\section{Consent for publication}

N/A.

\section{Competing interests}

The authors declare that they have no competing interests.

\section{Author details}

${ }^{1}$ College of Medicine Nursing and Health Sciences, National University of Ireland Galway, Galway, Ireland. 'Diabetic Day Centre, Galway University Hospital, Galway , Ireland. ${ }^{3}$ Department of Medicine, Brigham and Women's Hospital, Harvard Medical School, MA, Boston, USA. 
Received: 10 January 2020 Accepted: 3 July 2020

Published online: 18 July 2020

\section{References}

1. Metzger BE, Lowe LP, Dyer AR, Trimble ER, Chaovarindr U, Coustan DR, et al. Hyperglycemia and adverse pregnancy outcomes. N Engl J Med. 2008; 358(19):1991-2002.

2. Lowe WL, Scholtens DM, Kuang A, Linder B, Lawrence JM, Lebenthal Y, et al. Hyperglycemia and Adverse Pregnancy Outcome Follow-up Study (HAPO FUS): Maternal Gestational Diabetes and Childhood Glucose Metabolism. Diabetes Care. 2019:42(3):372.

3. Lowe WL, Scholtens DM, Lowe LP, Kuang A, Nodzenski M, Talbot O, et al. Association of Gestational Diabetes With Maternal Disorders of Glucose Metabolism and Childhood Adiposity. JAMA. 2018;320(10):1005-16.

4. Metzger BE. Long-term outcomes in mothers diagnosed with gestational diabetes mellitus and their offspring. Clin Obstet Gynecol. 2007;50(4):972-9.

5. Zera CA, Seely EW. Diabetes. Treatment of gestational diabetes reduces obstetric morbidity. Nat Rev Endocrinol. 2010;6(2):69-70.

6. Landon MB, Spong CY, Thom E, Carpenter MW, Ramin SM, Casey B, et al. A multicenter, randomized trial of treatment for mild gestational diabetes. $\mathrm{N}$ Engl J Med. 2009;361(14):1339-48.

7. Crowther CA, Hiller JE, Moss JR, McPhee AJ, Jeffries WS, Robinson JS, et al. Effect of treatment of gestational diabetes mellitus on pregnancy outcomes. N Engl J Med. 2005;352(24):2477-86.

8. Li-Zhen L, Yun X, Xiao-Dong Z, Shu-Bin H, Zi-Lian W, Adrian Sandra D, et al. Evaluation of guidelines on the screening and diagnosis of gestational diabetes mellitus: systematic review. BMJ Open. 2019;9(5):e023014.

9. Moses RG, Cheung NW. Point: Universal screening for gestational diabetes mellitus. Diabetes Care. 2009;32(7):1349-51.

10. Catalano PM, Avallone DA, Drago NM, Amini SB. Reproducibility of the oral glucose tolerance test in pregnant women. Am J Obstet Gynecol. 1993; 169(4):874-81.

11. Agarwal MM, Punnose J, Dhatt GS. Gestational diabetes: problems associated with the oral glucose tolerance test. Diabetes Res Clin Pract. 2004;63(1):73-4.

12. Sacks DA, Hadden DR, Maresh M, Deerochanawong C, Dyer AR, Metzger BE, et al. Frequency of gestational diabetes mellitus at collaborating centers based on IADPSG consensus panel-recommended criteria: the Hyperglycemia and Adverse Pregnancy Outcome (HAPO) Study. Diabetes Care. 2012;35(3):526-8.

13. Davies CS, Harris CL, Morgan BP. Glycation of CD59 impairs complement regulation on erythrocytes from diabetic subjects. Immunology. 2005;114(2): 280-6.

14. Meri S, Waldmann H, Lachmann PJ. Distribution of protectin (CD59), a complement membrane attack inhibitor, in normal human tissues. Lab Invest. 1991;65(5):532-7.

15. Yu J, Abagyan R, Dong S, Gilbert A, Nussenzweig V, Tomlinson S. Mapping the active site of CD59. J Exp Med. 1997;185(4):745-53.

16. Lynch AM, Salmon JE. Dysregulated complement activation as a common pathway of injury in preeclampsia and other pregnancy complications. Placenta. 2010;31(7):561-7.

17. Hoffman MC, Rumer KK, Kramer A, Lynch AM, Winn VD. Maternal and feta alternative complement pathway activation in early severe preeclampsia. Am J Reprod Immunol. 2014;71(1):55-60.

18. Buurma A, Cohen D, Veraar K, Schonkeren D, Claas FH, Bruijn JA, et al. Preeclampsia is characterized by placental complement dysregulation. Hypertension. 2012;60(5):1332-7.

19. Girardi G. Complement activation, a threat to pregnancy. Semin Immunopathol. 2018;40(1):103-11.

20. Girardi G, Yarilin D, Thurman JM, Holers VM, Salmon JE. Complement activation induces dysregulation of angiogenic factors and causes fetal rejection and growth restriction. J Exp Med. 2006;203(9):2165-75.

21. Salmon JE, Girardi G, Holers VM. Complement activation as a mediator of antiphospholipid antibody induced pregnancy loss and thrombosis. Ann Rheum Dis. 2002;61(Suppl 2):ii46-50.

22. Girardi G, Bulla R, Salmon JE, Tedesco F. The complement system in the pathophysiology of pregnancy. Mol Immunol. 2006;43(1-2):68-77.

23. Ghosh P, Luque-Fernandez MA, Vaidya A, Ma D, Sahoo R, Chorev M, et al. Plasma Glycated CD59, a Novel Biomarker for Detection of PregnancyInduced Glucose Intolerance. Diabetes Care. 2017;40(7):981-4.
24. O'Sullivan EP, Avalos G, O'Reilly M, Dennedy MC, Gaffney G, Dunne FP, et al. Atlantic DIP: the prevalence and consequences of gestational diabetes in Ireland. Ir Med J. 2012;105(5 Suppl):13-5.

25. Noctor E, Crowe C, Carmody LA, Kirwan B, O'Dea A, Glynn LG, et al. ATLANT IC-DIP: prevalence of metabolic syndrome and insulin resistance in women with previous gestational diabetes mellitus by International Association of Diabetes in Pregnancy Study Groups criteria. Acta Diabetol. 2015;52(1):15360.

26. Pepe MS. Evaluating technologies for classification and prediction in medicine. Stat Med. 2005;24(24):3687-96.

27. Brownlee M. The pathobiology of diabetic complications: a unifying mechanism. Diabetes. 2005;54(6):1615-25.

28. Acosta J, Hettinga J, Flückiger R, Krumrei N, Goldfine A, Angarita L, et al. Molecular basis for a link between complement and the vascular complications of diabetes. Proc Natl Acad Sci U S A. 2000;97(10):5450-5.

29. Flyvbjerg A. Diabetic angiopathy, the complement system and the tumor necrosis factor superfamily. Nat Rev Endocrinol. 2010;6(2):94-101.

30. Mellbin LG, Bjerre M, Thiel S, Hansen TK. Complement activation and prognosis in patients with type 2 diabetes and myocardial infarction: a report from the DIGAMI 2 trial. Diabetes Care. 2012;35(4):911-7.

31. Qin X, Goldfine A, Krumrei N, Grubissich L, Acosta J, Chorev M, et al. Glycation inactivation of the complement regulatory protein CD59: a possible role in the pathogenesis of the vascular complications of human diabetes. Diabetes. 2004;53(10):2653-61.

32. Meri S, Lehto T, Sutton CW, Tyynelä J, Baumann M. Structural composition and functional characterization of soluble CD59: heterogeneity of the oligosaccharide and glycophosphoinositol (GPI) anchor revealed by laserdesorption mass spectrometric analysis. Biochem J. 1996;316(Pt 3):923-35.

33. Lehto $T$, Honkanen $E$, Teppo AM, Meri S. Urinary excretion of protectin (CD59), complement SC5b-9 and cytokines in membranous glomerulonephritis. Kidney Int. 1995;47(5):1403-11.

34. Väkevä A, Lehto T, Takala A, Meri S. Detection of a soluble form of the complement membrane attack complex inhibitor CD59 in plasma after acute myocardial infarction. Scand J Immunol. 2000;52(4):411-4.

35. Ghosh P, Vaidya A, Sahoo R, Goldfine A, Herring N, Bry L, et al. Glycation of the complement regulatory protein CD59 is a novel biomarker for glucose handling in humans. J Clin Endocrinol Metab. 2014;99(6):E999-1006.

36. Ghosh P, Sahoo R, Vaidya A, Cantel S, Kavishwar A, Goldfine A, et al. A specific and sensitive assay for blood levels of glycated CD59: a novel biomarker for diabetes. Am J Hematol. 2013;88(8):670-6.

37. Halperin J, Dongdong M, Luque-Fernandez MA, Bogdanet D, Desoye G, Dunne F. Plasma Glycated CD59 (pGCD59) Predicts Early Gestational Diabetes (GDM) and Abnormal Neonatal Outcomes: DALI Study Secondary Analysis. American Diabetes Association; June 2019; San Francisco: Diabetes; 2019

\section{Publisher's Note}

Springer Nature remains neutral with regard to jurisdictional claims in published maps and institutional affiliations.

Ready to submit your research? Choose BMC and benefit from:

- fast, convenient online submission

- thorough peer review by experienced researchers in your field

- rapid publication on acceptance

- support for research data, including large and complex data types

- gold Open Access which fosters wider collaboration and increased citations

- maximum visibility for your research: over $100 \mathrm{M}$ website views per year

At $\mathrm{BMC}$, research is always in progress.

Learn more biomedcentral.com/submissions 